\title{
Economic weights of somatic cell score in dairy sheep
}

\author{
A. Legarra ${ }^{1 \dagger}$, M. Ramón ${ }^{2}$, E. Ugarte ${ }^{1}$, M. D. Pérez-Guzmán² and J. Arranz ${ }^{1}$ \\ ${ }^{1}$ NEIKER, Basque Institute of Agricultural Research and Development, Apartado 46, Vitoria-Gasteiz, 01080 Spain; ${ }^{2}$ CERSYRA, Avenida del vino s/n, 13.300, \\ Valdepeñas, Ciudad Real, Spain
}

(Received 17 March 2006; Accepted 9 November 2006)

\begin{abstract}
The economic weights for somatic cell score (SCS) have been calculated using profit functions. Economic data were collected in the Latxa breed. Three aspects have been considered: bulk tank milk payment, veterinary treatments due to high SCS, and culling. All of them are non-linear profit functions. Milk payment is based on the sum of the log-normal distributions of somatic cell count, and veterinary treatments on the probability of subclinical mastitis, which is inferred when individual SCS surpass some threshold. Both functions lead to non-standard distributions. The derivatives of the profit function were computed numerically. Culling was computed by assuming that a conceptual trait culled by mastitis (CBM) is genetically correlated to SCS. The economic weight considers the increase in the breeding value of CBM correlated to an increase in the breeding value of SCS, assuming genetic correlations ranging from 0 to 0.9. The relevance of the economic weights for selection purposes was checked by the estimation of genetic gains for milk yield and SCS under several scenarios of genetic parameters and economic weights. The overall economic weights for SCS range from -2.6 to $-9.5 €$ per point of SCS, with an average of $-4 €$ per point of SCS, depending on the expected average SCS of the flock. The economic weight is higher around the thresholds for payment policies. Economic weights did not change greatly with other assumptions. The estimated genetic gains with economic weights of $0.83 €$ per I of milk yield and $-4 €$ per point of SCS, assuming a genetic correlation of -0.30 , were 3.85 I and -0.031 SCS per year, with an associated increase in profit of $3.32 €$. This represents a very small increase in profit (about 1\%) relative to selecting only for milk yield. Other situations (increased economic weights, different genetic correlations) produced similar genetic gains and changes in profit. A desired-gains index reduced the increase in profit by $3 \%$, although it could be greater depending on the genetic parameters. It is concluded that the inclusion of SCS in dairy sheep breeding programs is of low economic relevance and recommended only if recording is inexpensive or for animal welfare concerns.
\end{abstract}

Keywords: dairy ewes, mastitis, profits, selection, somatic cell count.

\section{Introduction}

In dairy sheep breeding schemes, the objectives of selection and the merit index have often been set up in an empirical way, forced by the genetic changes observed in a population (Barillet et al., 1986), or by a desire of including traits considered as useful in the breeding programmes. One of the traits to be improved in dairy sheep is the somatic cell count (SCC) of the milk (Rupp et al., 2003), as an indicator of mastitis and as a trait influencing milk quality. However, there are no published estimates of the economic impact of the genetic improvement of SCC in dairy sheep production, which is a key aspect for the decision process leading to its inclusion in the selection objectives

\footnotetext{
${ }^{\dagger}$ Present address: Station d'Amélioration Génétique des Animaux, Département de Génétique Animale, Institut Nationale de la Recherche Agronomique, BP 52627, 31326 Castanet Tolosan Cedex, France. E-mail: legarra@toulouse.inra.fr
}

and criteria. The purpose of this work is to compute the economic weight of the related trait somatic cell score in dairy sheep.

\section{Traits}

Mastitis. Mastitis is one of the main diseases in sheep. As such, it is of high economic importance in dairy sheep production, because it leads to lowered production, culling of animals, and poor milk quality. According to Bergonier and Berthelot (2003), "the term 'mastitis' means udder inflammation, whatever the origin, severity and evolution", and "clinical mastitis is characterised by general signs (fever, anorexia, weakness, coma, etc.), or only local signs (udder inflammation and oedema, gangrena, asymmetry, sclerosis, abscesses, etc.) and functional signs (macroscopic or quantitative modifications of milk production). Subclinical mastitis is characterised by quantitative and qualitative functional modifications (especially an increase 
in somatic cell count)". Most mastitis is of infectious origin, with different pathogens and forms involved. For recent reviews on mastitis in dairy sheep, see Bergonier and Berthelot (2003) and Bergonier et al. (2003). In this work, we will not consider mastitis itself because subclinical mastitis status of the ewe can not be directly determined. Rather, subclinical mastitis it is usually inferred from the SCC (Bergonier and Berthelot, 2003).

Somatic cell count and somatic cell score. In dairy sheep, SCC is an indirect measure of subclinical mastitis infection. SCC is currently recorded in several milk recording schemes in dairy sheep, either systematically or experimentally (Astruc et al., 2004). The usual unit of expression of SCC is counts per millilitre. SCC shows a highly skewed distribution, which makes statistical analysis and the use of common selection tools (selection indices, BLUP) awkward. Thus, it is common to use a logarithmic transformation to achieve normality, with the new variable called somatic cell score (SCS).

Assuming a normal distribution for SCS assumes a log-normal distribution (e.g. Kendall and Stuart, 1963) for SCC. A log-normal distribution is the distribution of a variable whose logarithm is distributed as a normal. If variable $x$ follows a normal distribution, variable $y=e^{x}$ follows a log-normal distribution. The mean of the distribution of $y$ is:

$$
E(y)=\exp \left(\mu_{x}+\frac{\sigma_{x}^{2}}{2}\right),
$$

where $\mu_{x}$ and $\sigma_{x}^{2}$ are the mean and variance of $x$. The variance of $y$ is:

$$
\operatorname{Var}(y)=\exp \left(2 \mu_{x}+\sigma_{x}^{2}\right) \exp \left(\sigma_{x}^{2}-1\right) .
$$

These properties are of interest for the following sections.

The most used logarithmic transformation was proposed by Ali and Shook (1980), as follows: $S C S=\log _{2}$ $\left(S C C / 10^{5}\right)+3$. The logarithm is expressed in base two, which means that an increase of one in the SCS scale implies doubling in the SCC scale. Many studies exist on the genetic background of SCS in dairy sheep; for references, see Rupp et al. (2003). The economic weight will be therefore calculated on the Ali and Shook (1980) SCS scale, as the simplest one, and the scale in which estimated breeding values are calculated and presented. Transformation to other logarithmic scales is straightforward considering the ratio between the scales.

\section{Material and methods}

\section{Economics of SCC in dairy sheep}

We have considered five different economic consequences of the genetic improvement of SCS and therefore of SCC:

Loss of milk during lactation. The loss has been described in the Latxa dairy sheep by Romeo and Ziluaga (1998), to be about $40 \mathrm{ml} /$ day per 1 - point increase of SCS.
However, including this loss as related to genetics of SCS would cause the so-called double-counting (Groen et al., 1997a), as the genetic determinism of milk yield is already accounted for by considering the trait milk yield in the selection criteria. If there is an episode of severe mastitis, sheep is dried and its record is not considered for genetic evaluation, causing some bias, but clinical mastitis is difficult to relate to SCS. For completion, we point that the economic loss would be the loss in milk weighted by the price of the milk ( $0.92 €$ perl, as shown later). If the lactation length is 120 days, the value of the lost milk due to increase in SCS would be about $4.42 €$ per point of SCS.

Lower milk prices. As in cattle, the policy of dairy industries is to penalise milk prices if the SCS of the bulk tank milk within a certain period exceeds certain thresholds. There are a few penalisation systems depending on the factory, but most are very similar. We have chosen one of them which consists in paying, in relation to the standard price, $+0.018 €$ perl if the SCC of the bulk milk is lower than 300000 ; or, $-0.012 €$ perl if the SCC is higher than 750000 . This affects those farms who sell milk. There also other farms who produce cheese for sale.

Diminution of cheese yield and quality. This diminution is known (e.g. Jaeggi et al., 2003; Albenzio et al., 2004) but very hard to quantify and for this reason we ignored it. There are two types of farms in the Latxa breed, according to their final product: cheese-farmers (those who elaborate home-made cheese and sell it), and milkfarmers (those who sell milk to cheese factories). This would affect cheese-maker farms, for which we will not be able to predict an economic weight for this concept. If penalties in milk price are according to losses in cheese yield in the factories, the economic weight should be similar.

Veterinary treatment. When a ewe is considered to be infected and if infection is not too severe (subclinical mastitis), the veterinary treatment consists of inoculation with antibiotic and a period of suppression of milk of about three days, in which milk can not be sold or used. The cost of treatment oscillates from 1.20 to $2 €$; we retained a cost of $2 €$ to be conservative. The suppressed milk was considered to be three times the daily average production, which is around $800 \mathrm{ml}$. This makes $2.4 \mathrm{I}$ of milk, which, multiplied by a milk price of $0.92 €$ perl (Legarra et al., 2007) makes a total of $4.21 €$ per treatment.

Detection of mastitis status depends on level of SCC. Romeo et al. (1998) set the thresholds for the Latxa breed in 140000 (doubtful) and 340000 (infected). In practice, a rule of thumb is to consider a sheep to be infected if the SCC of one test day (TD) surpasses 200000 . This rule has been used in this work. Other rules (e.g. dynamic rules, which consider the change of SCC during lactations) and 
thresholds exist for different breeds (Bergonier and Berthelot, 2003). They are rather dependent on management systems and prevalence of mastitis in the studied flocks.

Lower ability, leading to culling. One of the reasons for culling in dairy sheep is a high susceptibility to mastitis, as seen by recurrent clinical or subclinical mastitis problems. As explained by Barillet et al. (2001), symptoms of clinical mastitis are modification of the colour or consistency of the milk, hot swollen or painful udders, positives at the California mastitis test, and udder abnormalities. This is the second most important reason for culling (after low milk production) in the experimental Lacaune flock of La Fage (Barillet et al., 2001). Recurrent high SCC is also cause of culling, but less frequent. We consider that this culling is involuntary, as it is forced by the presence of mastitis and not by the concept that the replacement ewe will be more profitable than the replaced one (which is the case for production traits).

Therefore, among all the economic consequences of high SCS, we have retained three: (a) the change in milk prices, (b) the cost of veterinary treatment and (c) involuntary culling due to mastitis. In setting up these economic consequences, we are assuming a management of the flock which is quite typical in the Latxa breed. Other farms or breeds may have different managements, e.g. reducing the number of drying-off treatments if there is a reduction in SCC, not treating ill animals, or using different culling strategies.

\section{Profit functions}

Separate profit functions were calculated for each of the three economical consequences of SCC presented above. Two of them are non-linear, as they depend on the probability of SCC, or SCS, or some aggregate trait, exceeding certain thresholds; therefore, the economic weight depends on the actual average of the population and the environmental variability of the trait. Analogous cases were presented by Hovenier et al. (1993), Colleau and Le Bihan-Duval (1995), and Veerkamp et al. (1998).

Milk payment. Penalties exist for milk payments beyond certain thresholds of SCC in bulk milk tank ( $\mathrm{SCC}_{\text {flock milk}}$ ). However, this not an individual measure, but an aggregate of many SCC phenotypes, which follow log-normal distributions. This poses some problems, as discussed by Veerkamp et al. (1998). The effect of changing the genetic background of one animal (or all the flock) is the average of a sum of log-normal distributions, which, moreover, will depend on present averages in the flock. Veerkamp et al. (1998) solved this problem by empirical simulation, sampling SCS records from the milk recording data.

The procedure is very similar to that discussed by Colleau and Le Bihan-Duval (1995), and presented in more detail by Dekkers (2003). Let all animals in the flock have an (individual) mean in the SCS scale of $\mu_{S C S}$ being dependent of environmental (management) or other (genetic) effects. At the flock level, the expectation of SCC in the flock (i.e. the SCC in flock bulk tank milk) can be calculated, by the features of the log-normal distribution, as

$$
E\left(S C C_{\text {flock milk }}\right)=\exp \left(\mu_{S C S}+\frac{\sigma_{S C S}^{2}}{2}\right),
$$

where $\sigma_{S C S}^{2}$ is the variability between subjects at the SCS scale, in this case, sheep composing the flock. The total variability implied in $\sigma_{S C S}^{2}$ is the sum of additive genetic, permanent environmental effect and residual variances. This assumes that all animals in the flock have the same production and therefore the average can be computed without accounting for milk yield. An association between SCC and milk yield could be considered, but the methodology would become more complex and the association is weak (genetic correlation estimates range from -0.20 to 0.10 ).

As we have shown, the expectation of the SCC of the flock bulk tank milk depends on the mean and variance of individual SCS. As such, this expression implies that $S C C_{\text {flock milk }}$ is constant, given those parameters. However, in practice there will be some variation (e.g. in different milkings) due to the fact that the number of ewes being milked $(n)$ is finite. Therefore, the variable $S C C_{\text {flock milk }}$ follows the probability distribution of the average of $n$ lognormal distributions. Let call this distribution $f\left(S C C_{\text {flock milk }}\right)$ which depends on $n, \mu_{S C S}$ and $\sigma_{S C S}^{2}$. There are also other sources of variation as lactation stage or parity that will not be considered here.

For a flock with given $\mu_{S C S}$ and $\sigma_{S C S}^{2}$, the variable $S C C_{\text {flock milk }}$ will be placed sometimes (say, in different milkings) under a given payment threshold and sometimes under another one. This implies different milk prices, with some associated average profit $P$. Hence, for an increase $d$ in $\mu_{S C S}$ the average profit $P$ will change in a quantity $c$. The economic weight of an increase in $\mu_{S C S}$ will be

$$
\frac{\partial P}{\partial \mu_{s C S}}
$$

evaluated at $\mu_{S C s}$. The average profit, $P$, can be computed as

$$
P=\sum_{i=1}^{m} p_{i} w_{i}
$$

where $m$ is the number of price categories and $p_{i}$ is the proportion in category $i$. The term $p_{i}$ can be calculated as the cumulative density function of $f\left(S C C_{\text {flock milk }}\right)$, that is:

$$
p_{i}=\int_{t(i-1)}^{t(i)} f\left(S C C_{f l o c k ~ m i l k}\right),
$$

where $t(i)$ is the upper payment threshold for the $i-$ th category (first and last threshold are zero and infinity). Colleau and Le Bihan-Duval (1995), and Dekkers (2003), could go a step further to avoid the computation of the 
derivatives and the cumulative density function, applying Leibnitz's rule to cancel out integrals and derivatives. However, applying the rule in this case was not possible. We computed the profit function by the Fenton-Wilkinson approximation and the derivatives by numerical methods, as explained in the Appendix. We assumed 150 animals being milked at the same time.

Veterinary treatment. The procedure is very similar to that discussed by Colleau and Le Bihan-Duval (1995), and presented in more detail by Dekkers (2003), and it is as follows:

Assume that an animal has an expectation for SCS of $\mu_{S C s .}$ On repeated measures of its phenotype, SCC will be expressed as a log-normal distribution, or, equivalently, SCS will be expressed as a normal distribution with mean $\mu_{S C S}$ and variance $\sigma_{S C S}^{2}$. Suppose that the sheep is treated if its SCS is over some threshold $t$. This value is the AliShook transformation of the threshold in the SCC scale, which we set to 200000 .

The percentage of treatments, $p$, can be calculated according to this distribution, and it will be:

$$
p(\text { treatment })=p(S C C>t)=\int_{t}^{+\infty} N\left(x \mid \mu_{S C S}, \sigma_{S C S}^{2}\right) d x
$$

where $N\left(x \mid \mu_{S C S} \sigma_{S C S}^{2}\right)$ is the normal distribution of $x$.

Let consider an increase in the breeding value, $d$. The new percentage of treatments, $p_{1}$, will be:

$$
p(\text { treatment })=p(S C C>t)=\int_{t}^{+\infty} N\left(x \mid \mu_{S C S}+d, \sigma_{S C S}^{2}\right) \mathrm{d} x .
$$

Accordingly, the economic weight of an increase of $d$ will be $\left(p_{1}-p\right) c$, where $c$ is the cost of the treatment.

However, this is valid as far as one TD analysis is considered. On average, four TD are analysed within a given lactation. For a given lactation, they can be considered as the same trait, the only variation due to within-lactation variance. We can also consider that, once a sheep has been medically treated, there are no more outcomes of subclinical mastitis for that lactation. Therefore the risk of having an episode of subclinical mastitis in a given TD is:

$p$ (having subclinical mastitis) $\times p($ not having it previously), the last probability being

$$
1-p(S C C>t)=1-\int_{t}^{+\infty} N\left(x \mid \mu_{S C S}, \sigma_{S C S}^{2}\right) \mathrm{d} x .
$$

These probabilities have to be added for the four TDs considered, so that

$$
p(\text { detecting subclinical mastitis in the lactation })=\sum_{i=1}^{4} p_{i},
$$

where $p_{i}=\left(1-p_{i-1}\right) p(S C C>t)$ and

$$
p(S C C>t)=\int_{t}^{+\infty} N\left(x \mid \mu_{S C S}, \sigma_{S C S}^{2}\right) d x .
$$

Thus, the probability of an animal being infected at one of the four TDs in the lactation is a non-standard probability distribution. Derivatives of this distribution were computed by numerical methods, and weighted by the economic relevance for each category to obtain the derivatives of the profit function and the economic weight of SCS for veterinary treatment.

Involuntary culling. To include this concept in the profit function, the (genetic) relationship between clinical mastitis and SCC, and the culling policies for clinical mastitis or SCC should be known. The first one is not; in fact, as pointed out by Barillet et al. (2001), clinical mastitis usually occurs early in lactation, when SCC are not available. The culling policies are also poorly known.

To explore the bounds of the economic weight for this concept, we assumed a model based on genetic correlations. We define a conceptual trait 'culled by mastitis' (CBM), with a value of 1 if the ewe is culled because of mastitis, and zero otherwise. According to Barillet et al. (2001), this event is more related to clinical mastitis (93\%) than to high SCC (7\%), so we will assume CBM to be fairly similar to clinical mastitis. The genetic relationship between CBM and SCS will be defined by a more or less strong genetic correlation.

In dairy cattle, a value of 0.7 is a usual estimate of the genetic correlation between clinical mastitis and SCS (Carlén et al., 2004). Mastitis in small ruminants is quite different from cattle (Bergonier et al., 2003), and lower correlations between CBM and SCS have to be considered. Different values $(0,0.5,0.7,0.9)$ of the genetic correlation between CBM and SCS were therefore tested.

The incidence of CBM thus may be related to the genetic background for SCS. According to Barillet et al. (2001), frequency of $\mathrm{CBM}=1$ (culling) was $5.3 \%$ in first lactation; we assumed it to be constant for all lactations. This is a rather strong assumption but there is no other information available. This value was assumed to hold for all levels of SCC of the flock, because Barillet et al. (2001) observed the same incidence for the two divergent lines in the La Fage experimental flock, which substantially differ in the average SCC. Phenotypic variance of the binomial distribution of CBM is therefore $0.053 \times(1-0.053)=0.05$. Genetic variance, assuming a heritability of 0.05 , would be 0.0025 . Therefore, the regression of the breeding value of CBM on the breeding value of SCS is:

$$
u_{C B M}=u_{S C S} \frac{\sigma_{C B M, S C S}}{\sigma_{S C S}^{2}}
$$

where $\sigma_{\mathrm{CBM}, \mathrm{SCS}}$ is the genetic covariance between both traits and $\sigma_{\text {SCS }}^{2}$ is the genetic variance for SCS which was assumed to be 0.20 (Legarra and Ugarte, 2005). Including 
the genetic correlation in the formula and substituting the variances gives:

$$
u_{C B M}=0.118 r_{g} u_{S C S}
$$

where $r_{g}$ is the genetic correlation between CBM and SCS. Therefore a genetic increase of one unit of SCS increases CBM in 0.12 times the genetic correlation; 0 for null genetic correlation, $5 \%$ for a genetic correlation of $0.5,7.8 \%$ for a genetic correlation of 0.7 , and $10.6 \%$ for a genetic correlation of 0.9 .

Cost of culling is hard to compute in the presence of quota or when culling is voluntary (e.g. Colleau and Le Bihan-Duval, 1995; Groen et al., 1997b). This is not the case, as culling is involuntary and there is no quota. No correction for the different production in different lactations was applied. Economic data was taken from Legarra et al. (2007). The average age of the ewe culled by mastitis was calculated considering the probabilities of being culled by mastitis $(0.053)$ or by any other reason (this was set to 0.13 to mimic a culling rate of 0.18 ). The result is 2.76 lactations. The cost of the replacement ewe is the cost of raising the hogget $(52 €)$, divided by 5.58 lactations, multiplied by the 2.82 lactations in which the new ewe is considered to replace the culled ewe. This gives an average cost of involuntary culling of $26 €$ per culled sheep. Notice that the cost of $52 €$ is rather low in relation to free market price for hoggets (which can be two or three times that amount). In the free market what is paid is also the cost of opportunity of having a genetically good flock and the installations in which to keep hoggets for sale. The $52 €$ have to be considered as the increased cost originated to the farm by breeding one more replacement hogget.

The former expression for the increased frequency of culled ewes (CBM), multiplied by a cost of $26 €$, gives the economic weight, i.e. the change in profit due to a genetic change in SCS.

Overall. The overall economic weight was calculated as the sum of the three components already presented.

\section{Parameters}

We have calculated the economic weights for different values of $\mu_{S C S}$ (average SCS of the ewe), which in turn correspond to values of average SCC of the flock between 50000 and $1000000 \mathrm{SCC}$ per ml, which is the usual range of values in dairy sheep. The values of the variances (Legarra and Ugarte, 2005) are: additive genetic variance $=0.20$, permanent environmental variance $=0.18$, residual variance $=1.16$.

\section{Sensitivity analysis}

To check the effect of the different thresholds, payment systems and prices, a few parameters were changed: (a) penalties for milk flock SCC were doubled; (b) thresholds for milk flock SCC were made more stringent (200000 and 500000 SCC per $\mathrm{ml}$ ); (c) the number of sheep being milked was assumed to be 50 (c1) or 500 (c2); (d) cost of veterinary treatment was changed in $+50 \%$ (d1) and $-50 \%$ (d2); (e) threshold for veterinary treatment was changed to 100000 (e1) and to 300000 (e2) SCC per ml.

The effect of different genetic correlations between SCS and CBM has already been noted. Increasing the cost of replacement would increase the economic weight in the same proportion.

\section{Genetic gains}

Theoretical genetic gains were calculated using selection index theory assuming a simplified breeding scheme considering different pathways of selection (see Legarra et al. (2007) for details). Two traits, milk yield and SCS, were considered as sources of information for the selection indexes. Different selection indices were used based on different economic weights. The economic weight for milk yield was calculated by Legarra et al. (2007). For SCS, we used two of the economic weights calculated in this study for SCS, as well as a desired gains pseudo-economic weight that will be shown later. Genetic gains were also calculated when the selection index only considers milk yield, to check the correlated responses for SCS. In the case of selection for milk yield, it was assumed that no information for SCS was used; this is according to a practical application (if SCS is not selected for, it is unlikely that it is used for genetic evaluation), and results in slightly lowered accuracies of the selection indexes.

Two scenarios were considered for the genetic parameters. Legarra and Ugarte (2005) estimated in the Latxa breed heritabilities of 0.21 for milk yield and 0.13 for SCS, and a (favourable) genetic correlation of -0.30 . Rupp et al. (2003) estimated in the Lacaune breed a genetic correlation that turns out to be unfavourable (between 0.08 and 0.18). The pseudo-economic weight was calculated to fix, under these conditions, the genetic change of SCS to zero.

\section{Results}

\section{Economic weights}

Results are shown in Figure 1 for the case where the genetic correlation between SCS and CBM is 0.7. The economic weights due to culling are zero for a null genetic correlation, and $-1.38,-1.93$ and $-2.49 €$ for genetic correlations of $0.5,0.7$ and 0.9 respectively. This economic weight is constant at different levels of SCC of the flock. This is the major part of the overall economic weight unless the average SCC of the flock is close to a pricing threshold.

The absolute economic weight of SCS due to payment penalties is only relevant when the SCC level of the flock is close to the thresholds, because, at any other point, the probability of reaching any better payment threshold is almost null. In other words, when the average level of SCC is close to the thresholds, it is worth investing in genetic improvement of SCS because there is a high probability of milk being sold for a higher price. The absolute economic 


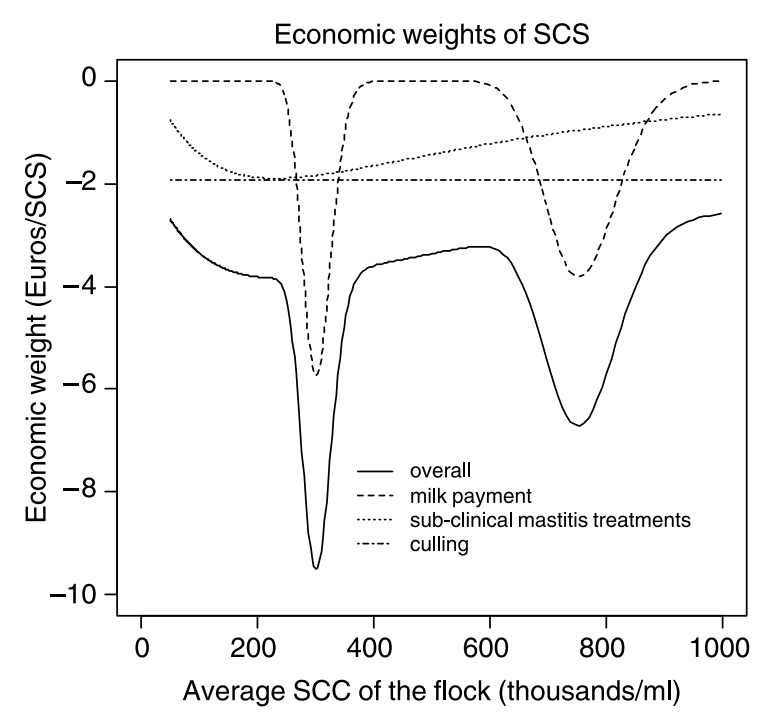

Figure 1 Overall economic weight of somatic cell score (SCS) and its components for different levels of somatic cell count (SCC) of the flock. The assumed genetic correlation between 'culling by mastitis' and SCS is 0.7 .

weight of subclinical mastitis treatments increases and then decreases. The reason is that, the closer we are to the threshold for treatment, the more treatments are saved by improving SCS. In other words, at low levels of SCC there are very few treatments to be saved, and at high levels of SCC all animals are theoretically infected and improving SCS does not save many treatments.

As a result, the overall economic weight of SCS is about -3 to $-4 €$ per point of SCS per productive ewe in the flock in the range of common values of SCC in dairy sheep, except when the average SCC of the flock is around the payment thresholds, when it decreases down to $-9.50 €$ per point of SCS (at 303000 counts per ml) and -6.71 (at 754000 counts per $\mathrm{ml}$ ).

\section{Sensitivity analysis}

The different curves of economic weights obtained varying the different assumptions are shown in Figure 2. Increasing the payment penalties increases the economic levels, which also affect a wider range of average SCC of the flock. Using more stringent levels shifts the curve of economic weight to the left. Changing the number of animals changes the value of the economic weight and its position. Decreasing the number of animals makes that, for an increase in SCC, there is a higher risk of reaching a different quality threshold for SCC flock averages farther than the thresholds. This, in turn, decreases the maximum absolute value of the economic weight. A change in costs or thresholds for treatments changes the economic weights, but not in a great manner.

\section{Genetic gains}

The two economic weights chosen for SCS were $-4 €$ per point of SCS, which is the average economic weight for SCS, and $-9.50 €$ per point of SCS, which is the maximum
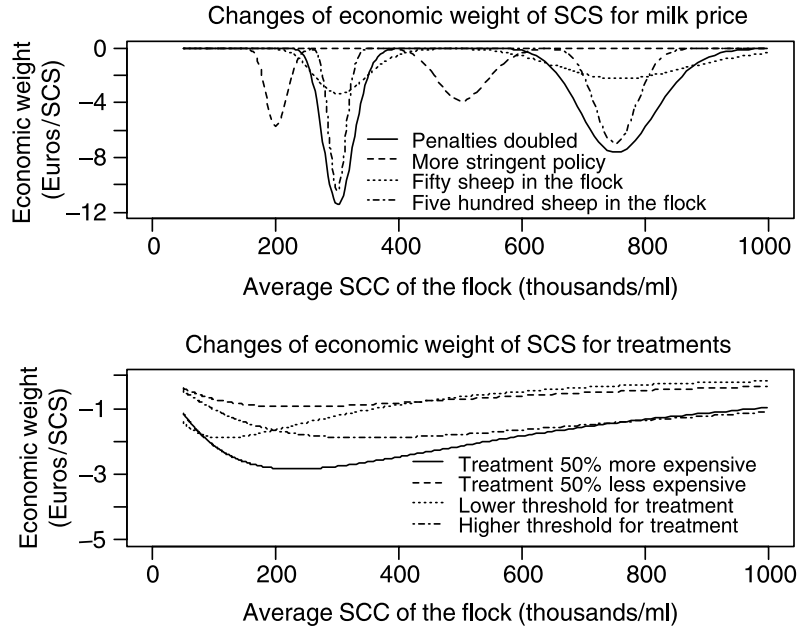

Figure 2 Sensitivity analysis of economic weights of somatic cell score (SCS) for different levels of somatic cell count (SCC) of the flock.

absolute value. The economic weight for milk yield was $0.83 €$ per I (median of economic weight in milk-selling farms (Legarra et al., 2007)).

Genetic gains per year and associated increases in profit are shown in Table 1. Inclusion of SCS in the selection scheme leads to increased profits of $0.9 \%$ if the economic value is $-4 €$ per point of SCS and $2.6 \%$ if the economic value is $-9.50 €$ per point of SCS. The genetic change for SCS and the increase in profit is small for all possible economic weights and genetic parameters.

Imagine that the aim of a breeding programme, facing the unfavourable genetic correlation between milk yield and SCS, were to fix the level of SCS. In the case of unfavourable genetic correlation, a vector of pseudo-economic weights to attain this objective was 0.83 for milk yield and -17.53 for SCC, for which the genetic gains are 3.66 for milk yield and 0 for SCS. These weights give four times more importance to SCC than the economic weights. The profit gain would decrease by $3 \%$ relative to the economically optimal set of weights ( $-4 €$ per point of SCS) or selecting for milk yield only. If the true genetic correlation is favourable, the decrease in profit gain is lower (around 2\%).

\section{Discussion}

The economic weight of SCC oscillates between -3 and $-4 €$ per point of SCS for most levels of SCC of the flock, with peaks close to the payment thresholds of about $-9 €$ per point of SCS. These are small figures for practical applications, as observed by the genetic gains, where the expected increase in profit ranges from $0.9 \%$ to $2.6 \%$.

Different alternatives present different economic weights; however, it seems likely that economic weight for SCS are bounded from -1 to $-10 €$ per point of SCS. Even in the higher bounds, the additional profit by including SCS in the breeding objective is not expected to be very high for two reasons: first, it is a low economic weight, as the unit of measure is rather high (a decrease 
Table 1 Genetic gains (per year) and increase in profit considering different economic weights and genetic correlation $\left(r_{g}\right)$ between milk yield and somatic cell score (SCS)

Scenario

\begin{tabular}{|c|c|c|}
\hline \multicolumn{2}{|c|}{ Genetic gain } & \multirow[b]{2}{*}{ Increase in profit (€ per sheep) } \\
\hline Milk yield (I) & SCS & \\
\hline \multicolumn{3}{|l|}{ of $\mathrm{SCS}^{\dagger}$} \\
\hline 3.80 & -0.037 & 3.50 \\
\hline 3.85 & 0.013 & 3.08 \\
\hline \multicolumn{3}{|l|}{ f SCS } \\
\hline 3.85 & -0.031 & $3.32^{\S}$ \\
\hline 3.91 & 0.021 & $3.16^{\S}$ \\
\hline \multicolumn{3}{|c|}{ er point of $\mathrm{SCS}^{\ddagger}$} \\
\hline 3.69 & -0.043 & $3.24^{\S}$ \\
\hline 3.66 & 0 & $3.04^{\S}$ \\
\hline 3.85 & -0.023 & $3.29^{\S}$ \\
\hline 3.85 & 0.014 & $3.14^{\S}$ \\
\hline
\end{tabular}

\begin{tabular}{|c|c|}
\hline \multicolumn{2}{|c|}{ Economic weight $=-9.50 €$ per point of $\mathrm{SCS}^{\dagger}$} \\
\hline$r_{g}=-0.30$ & 3.80 \\
\hline$r_{g}=0.18$ & 3.85 \\
\hline \multicolumn{2}{|c|}{ Economic weight $=-4.0 €$ per point of SCS } \\
\hline$r_{g}=-0.30$ & 3.85 \\
\hline$r_{g}=0.18$ & 3.91 \\
\hline \multicolumn{2}{|c|}{ Pseudo-economic weight $=-17.53 €$ per point of SCS } \\
\hline$r_{g}=-0.30$ & 3.69 \\
\hline$r_{g}=0.18$ & 3.66 \\
\hline \multicolumn{2}{|l|}{ Milk yield only } \\
\hline$r_{g}=-0.30$ & 3.85 \\
\hline$r_{g}=0.18$ & 3.85 \\
\hline
\end{tabular}

of one in SCS means halving SCC); second, because the heritability and the genetic variances are low, the genetic improvement is slow. Quota systems or animal welfare concerns would increase the economic weight of SCS.

The low economic impact is quite independent of the assumed genetic correlation between SCS and milk yield. These are still a matter of discussion, as estimates in the Lacaune breed are unfavourable, whereas estimates in the Spanish breeds are favourable (e.g. Rupp et al. (2003) and Legarra and Ugarte (2005) and references therein) but we have shown that for different estimates either economic weights or a desired gains index are adequate, the latter being slightly economically worse. Yet one needs to be careful not to set too strong a restriction for the change in SCS, because the risk of greatly diminishing gains for milk yield exists depending on the true genetic parameters.

Drawbacks of the study are: first, some assumptions were taken in order to define profit functions. These include culling policies, culling rates, and thresholds for treatment and payment. Therefore the final results have to be considered as an approximation, based mainly on the management made in Latxa flocks. Second, the economic weight of culling due to mastitis was considered by an indirect reasoning, namely, the hypothetical correlations between SCC and the animal being culled by mastitis. We have tried to check some of these assumptions through the sensitivity analysis, which shows no big practical differences in genetic improvement. At any rate, the assumptions proved to be useful to set the range of values for the overall economic weight. A much more detailed study on culling policies and relation between SCS, SCC, mastitis and survival in dairy sheep is needed, and this should be one direction for future research.

Including SCS in dairy sheep breeding objectives has to be considered with caution, given its low economic weight and slow genetic improvement. The major cost of genetic improvement of SCS will be the implementation of SCS recording, which is usually associated with milk composition recording. Milk composition recording is being implemented in several countries (Astruc et al., 2004). In those cases, it seems reasonable to consider the inclusion of SCS in the breeding program. At any rate, it seems a better strategy at the beginning to first reduce mastitis/SCS problems by management (good hygienic and milking practices, drying-off treatments), which is quicker and cheaper than selection (Bergonier et al., 2003). If changes in management are not useful anymore, selection might be a good option.

\section{Acknowledgements}

This work has been financed by project INIA RTA $-02-002-C 2$. A. Legarra was partly financed by INIA, Spain. Manuel Ramón thanks the research fellowship given by INIA, Spain. We are grateful for the information on veterinary treatments provided by Javier García, Ane Garziandia and Rafael San Sebastián. We thank the referees for helpful comments.

\section{References}

Albenzio M, Caroprese M, Santillo A, Marino R, Taibi L and Sevi A 2004. Effects of somatic cell count and stage of lactation on the plasmin activity and cheese-making properties of ewe milk. Journal of Dairy Science 87, 533-542.

Ali AKA and Shook GE 1980. An optimum transformation for somatic cell count in milk. Journal of Dairy Science 63, 487-490.

Astruc JM, Barillet F, Fioretti M, Gabina D, Gootwine E, Mavrogenis AP, Romberg FJ, Sanna SR and Stefanake E 2004. Report of the working group on milk recording on sheep. In Proceedings of the 34th ICAR session, Sousse, Tunisia, 30 May to 3 June. Available at www.icar.org

Barillet F, Elsen JM and Roussely M 1986. Optimization of a selection scheme for milk composition and yield in milking ewes: example of the Lacaune 
breed. In Proceedings of the third world congress on genetics applied to livestock production. IX, Lincoln, Nebraska, pp. 658-664.

Barillet F, Rupp R, Mignon-Grasteau S, Astruc JM and Jacquin M 2001. Genetic analysis for mastitis resistance and milk somatic cell score in French Lacaune dairy sheep. Genetics, Selection, Evolution 33, 397-415.

Bergonier D and Berthelot X 2003. New advances in epizootiology and control of ewe mastitis. Livestock Production Science 79, 1-6.

Bergonier $D$, de Crémoux R, Rupp R, Lagriffoul $G$ and Berthelot X 2003. Mastitis of dairy small ruminants. Veterinary Research 34, 689-716.

Carlén E, Strandberg E and Roth A 2004. Genetic parameters for clinical mastitis, somatic cell score, and production in the first three lactations of Swedish Holstein cows. Journal of Dairy Science 87, 3062-3070.

Cheney W and Kincaid D 1994. In Numerical mathematics and computing, 3rd ed. Brooks/Cole, Pacific Grove, California.

Colleau JJ and Le Bihan-Duval E 1995. A simulation study of selection methods to improve mastitis resistance of dairy cows. Journal of Dairy Science 78, 659-671.

Dekkers JCM 2003. In Design and economics of animal breeding strategies. Notes for summer short course, 14-18 July. lowa State University, Ames, lowa.

Groen AF, Steine T, Colleau JJ, Pedersen J, Pribyl J and Reinsch N 1997a. Economic values in dairy cattle breeding, with special reference to functional traits. Report of an EAAP-working group. Livestock Production Science 49, 1-21.

Groen AF, Van Arendonk JAM, Goddard ME and Vollema AR 1997b. On the use of opportunity costs in deriving the economic value of herd life. Animal Science 65, 335-341.

Hovenier R, Brascamp EW, Kanis E, Van der Werf JHJ and Wassenberg APAM 1993. Economic values of optimum traits: the example of meat quality in pigs. Journal of Animal Science 71, 1429-1433.

Jaeggi J, Govindasamy-Lucey S, Berger YM, Johnson ME, McKusick BC, Thomas DL and Wendorff WL 2003. Hard ewe's milk cheese manufactured from milk of three different groups of somatic cell counts. Journal of Dairy Science 86, 3082-3089.

Legarra A, Ramón M, Ugarte $E$ and Pérez-Guzmán MD 2007. Economic weights of fertility, prolificacy, milk yield and longevity in dairy sheep. Animal 1, 193-203.

Legarra A and Ugarte E 2005. Genetic parameters of udder traits, somatic cell score, and milk yield in Latxa sheep. Journal of Dairy Science 88, 2238-2245.

Kendall MG and Stuart A 1963. In The advanced theory of statistics, volume 1. Distribution theory, 2nd ed. Charles Griffin and Company Limited, London.

Romeo M, Ziluaga I and Arranz J 1998. [Evaluation of loss in yield due to mastitis and prevalence of infected animals in the flock in relation to bulk tank milk somatic cell count. New strategy of mastitis control using an immunologic booster.] In Jornada de controladores 1998. Neiker, Vitoria-Gasteiz, Spain.

Romeo M, Ziluaga I and Marco J 1998. Diagnosis in situ of mammary infection by palpation. California mastitis test and monitoring by somatic cell count. Ovis 59, 61-77.

Rupp R, Lagriffoul G, Astruc JM and Barillet F 2003. Genetic parameters for milk somatic cell scores and relationships with production traits in French Lacaune dairy sheep. Journal of Dairy Science 86, 1476-1481.

Veerkamp RF, Stott AW, Hill WG and Brotherstone S 1998. The economic value of somatic cell count payment schemes for UK dairy cattle breeding programmes. Animal Science 66, 293-298.

\section{Appendix}

Computation of derivatives of an average of log-normal variables

Let $x \sim \operatorname{Normal}\left(\mu_{x}, \sigma_{x}^{2}\right)$ and $y=\exp (x)$. The variable $y$ follows a log-normal distribution whose density can be computed as:

$$
p(y)=\frac{1}{y \sqrt{2 \pi \sigma_{x}^{2}}} \exp \left(-\frac{1}{2} \frac{\left(\log (y)-\mu_{x}\right)^{2}}{\sigma_{x}^{2}}\right) .
$$

The mean and variance of $y$ are:

$$
E(y)=\exp \left(\mu_{x}+\frac{\sigma_{x}^{2}}{2}\right) ; \operatorname{Var}(y)=\exp \left(2 \mu_{S C S}+\sigma_{S C S}^{2}\right)
$$

The distribution $f\left(S C C_{\text {flock milk }}\right)$ is the distribution of the average of a sum of $n$ variables (individual SCC of daily yields) which are log-normally distributed with parameters $\mu_{S C S}$ and $\sigma_{S C S}^{2}$. This distribution has not general closed form, but it is possible to use the so-called Fenton-Wilkinson approximation, which states that a sum of log-normal variables follows approximately another log-normal distribution, of exact mean

$$
\begin{aligned}
\mu_{S C C_{\text {flock milk }}} & =E\left(S C C_{\text {flock milk }}\right) \\
& =\sum_{i} w_{i} \exp \left(\mu_{S C S}+\frac{\sigma_{S C S}^{2}}{2}\right)
\end{aligned}
$$

and approximated variance:

$$
\begin{aligned}
\sigma_{S C C_{\text {flockmilk }}}^{2} & =\operatorname{Var}\left(S C C_{\text {flock milk }}\right) \\
& \approx \sum_{i} w_{i}^{2} \exp \left(2 \mu_{S C S}+\sigma_{S C S}^{2}\right),
\end{aligned}
$$

where $w_{i}$ are the weights for each variable; in this case, we assume that $w_{i}$ is constant and $w_{i}=1 / n$.

However, the density of the log-normal distribution of $\mathrm{SCC}_{\text {flock milk }}$ is parameterised in terms of the log of $\mathrm{SCC}_{\text {flock }}$ milk (let call it $\mathrm{LSCC}_{\text {flock milk}}$ ), which follows a normal distribution. To find the appropriate parameters, one can solve as follows:

$$
\begin{aligned}
\mu_{L S C C_{\text {flock milk }}} & =E\left(\log \left(S C C_{\text {flock milk }}\right)\right) \\
& =\log \left(\frac{\mu_{S C S_{\text {flock milk }}}^{2}}{\sqrt{\mu_{S C S_{\text {flock milk }}^{2}}+\sigma_{S C S_{\text {flock milk }}}^{2}}}\right) \\
\sigma_{L S C C_{\text {flock milk }}}^{2} & =\operatorname{Var}\left(\log \left(S C C_{\text {flock milk }}\right)\right) \\
& =\log \left(1+\frac{\sigma_{S C S_{\text {flock mik }}}^{2}}{\mu_{S C S_{\text {flock milk }}}^{2}}\right)
\end{aligned}
$$

From the log-normal density function defined by these parameters, it is possible to construct the average profit, due to payment policies, for any given values of $\mu_{S C S}$ and $\sigma_{S C S}^{2}$ by: first, solving the above equations; second, calculating the probabilities that the SCC of the flock bulk tank milk falls within two thresholds (using the cumulative density function by the $R$ function plnorm); and third, multiplying the probabilities by the respective penalties and the total milk per animal. The derivatives of this function -hence the economic weights- for different values of $\mu_{S C S}$ were calculated by numerical methods (interpolation polynomials, Cheney and Kincaid (1994)). 